\title{
Anomalous Transport Due to the Conformal Anomaly
}

\author{
M. N. Chernodub* \\ CNRS, Laboratoire de Mathématiques et Physique Théorique UMR 7350, Université de Tours, 37200 France \\ Soft Matter Physics Laboratory, Far Eastern Federal University, Sukhanova 8, Vladivostok, 690950, Russia and \\ Department of Physics and Astronomy, University of Gent, Krijgslaan 281, S9, B-9000 Gent, Belgium
}

(Dated: September 30, 2016)

\begin{abstract}
We show that the scale (conformal) anomaly in field theories leads to new anomalous transport effects that emerge in an external electromagnetic field in an inhomogeneous gravitational background. In inflating geometry the QED scale anomaly locally generates an electric current that flows in opposite direction with respect to background electric field (the scale electric effect). In a static spatially inhomogeneous gravitational background the dissipationless electric current flows transversely both to the magnetic field axis and to the gradient of the inhomogeneity (the scale magnetic effect). The anomalous currents are proportional to the beta function of the theory.
\end{abstract}

Anomalous transport phenomena emerge in systems with quantum anomalies that break certain classical symmetries and lead to nonconservation of associated (otherwise classically conserved) currents [1, 2]. For example, the axial symmetry of chiral (massless) fermions is broken by the axial anomaly that naturally leads to nonconservation of the axial current at the quantum level [3]:

$$
\partial_{\mu} j_{A}^{\mu}=\frac{e^{2}}{16 \pi^{2}} F_{\mu \nu} \widetilde{F}^{\mu \nu},
$$

where $F_{\mu \nu}=\partial_{\mu} A_{\nu}-\partial_{\nu} A_{\mu}$ is the field-strength tensor of an Abelian gauge field $A_{\mu}$ and $\widetilde{F}^{\mu \nu}=\frac{1}{2} \varepsilon^{\mu \nu \alpha \beta} F_{\alpha \beta}$.

The simplest anomalous transport laws induced by the axial anomaly (1) are the chiral separation effect (CSE) [4, 5] and the chiral magnetic effect (CME) [6, 7]:

$$
\boldsymbol{j}_{A}=\frac{\mu_{V}}{2 \pi^{2}} e \boldsymbol{B}, \quad \boldsymbol{j}_{V}=\frac{\mu_{A}}{2 \pi^{2}} e \boldsymbol{B},
$$

that generate, respectively, the axial current $j_{A}$ and the vector current $\boldsymbol{j}_{V}$ along the axis of the external magnetic field $\boldsymbol{B}$ in dense $\left(\mu_{V} \neq 0\right)$ and in chirally imbalanced $\left(\mu_{A} \neq 0\right)$ medium. The chemical potential $\mu_{V}$ and the chiral chemical potential $\mu_{A}$ are thermodynamically conjugated to the total charge density $j_{V}^{0}$ and to the axial charge density $j_{A}^{0}$, respectively.

The axial anomaly (1) is also responsible for the density-dependent contributions to the chiral vortical effects [8, 9] which generate vector and axial currents,

$$
\boldsymbol{j}_{V}=\frac{\mu_{V} \mu_{A}}{\pi^{2}} \boldsymbol{\Omega}, \quad \boldsymbol{j}_{A}=\left(\frac{T^{2}}{6}+\frac{\mu_{V}^{2}+\mu_{A}^{2}}{2 \pi^{2}}\right) \boldsymbol{\Omega},
$$

in chiral fluids that rotate with the angular velocity $\boldsymbol{\Omega}$. The temperature-dependent $T^{2}$ part of the rotationinduced axial current in Eq. (3) is a result of the mixed axial-gravitational anomaly [10]:

$$
\partial_{\mu} j_{A}^{\mu}=-\frac{1}{384 \pi^{2}} R_{\mu \nu \alpha \beta} \widetilde{R}^{\mu \nu \alpha \beta}
$$

where $\widetilde{R}^{\mu \nu \alpha \beta}$ is the Riemann curvature tensor of a curved space background and $\widetilde{R}^{\mu \nu \alpha \beta}=\frac{1}{2} \varepsilon^{\mu \nu \gamma \lambda} R_{\gamma \lambda}^{\alpha \beta}$. Despite that anomaly (4) is formulated in a curved background, the associated anomalous transport is realized in a flat space too. In the presence of electromagnetic field in a curved background the total divergence of the axial current is given by the sum of the right-hand sides of Eqs. (1) and (4).

The anomalous transport laws (2) and (3) are invariant under time inversion $T: t \rightarrow-t$. Since the entropy cannot decrease with time, the $T$ invariance implies that these anomalous currents correspond to reversible processes which do not generate entropy. In other words the anomalous transport laws are nondissipative phenomena. They play an increasingly important role both in condensed matter physics and in high energy physics [11, 12.

Besides the axial and mixed axial-gravitational anomalies, a class of physically interesting quantum field theories is also subjected to a scale anomaly ${ }^{1}$ which breaks classical scale invariance of the theory at the quantum level [3]. Since it seems now quite natural to think that at least some quantum anomalies may be associated with certain anomalous transport laws [1, 2, we would like to ask a natural question: does the conformal anomaly lead to a new anomalous transport law?

In this Letter we consider a simplest case of a $\mathrm{U}(1)$ gauge theory with one massless Dirac fermion field $\psi$ described by the following Lagrangian:

$$
\mathcal{L}=-\frac{1}{4} F_{\mu \nu} F^{\mu \nu}+\bar{\psi} i \not D \psi,
$$

where $\not D=\gamma^{\mu} D_{\mu}$ and $D_{\mu}=\partial_{\mu}+i e A_{\mu}$ is the covariant derivative. This theory does not involve any characteristic length or energy scale since its Lagrangian (5) possesses only dimensionless coupling $e$. Therefore at a classical level the massless electrodynamics (5) is invariant under redefinition of the absolute length or energy scales. The corresponding scale transformations are generated by the dilatation current:

$$
j_{D}^{\mu}=T^{\mu \nu} x_{\nu}
$$

\footnotetext{
1 Also known as the dilatation, trace, conformal or Weyl anomaly.
} 
where the (symmetric) energy-momentum tensor

$$
\begin{aligned}
T^{\mu \nu}= & -F^{\mu \alpha} F_{\alpha}^{\nu}+\frac{1}{4} \eta^{\mu \nu} F_{\alpha \beta} F^{\alpha \beta} \\
& +\frac{i}{2} \bar{\psi}\left(\gamma^{\mu} D^{\nu}+\gamma^{\nu} D^{\mu}\right) \psi-\eta^{\mu \nu} \bar{\psi} i \not D \psi,
\end{aligned}
$$

can be obtained by the variation of the action $S$ with respect to the background metric $g_{\mu \nu}$ :

$$
T^{\mu \nu}(x)=2 \frac{\delta S}{\delta g_{\mu \nu}(x)}, \quad S=\int d^{4} x \sqrt{-g} \mathcal{L},
$$

with $g=\operatorname{det}\left(g_{\mu \nu}\right)$. We restore the flat space-time metric $g^{\mu \nu} \rightarrow \eta^{\mu \nu}=\operatorname{diag}(+1,-1,-1,-1)$ after the variation.

Classically, the dilatation current (6) has zero divergence because the classical equations of motion imply

$$
\partial_{\mu} j_{D}^{\mu}=T_{\alpha}^{\alpha},
$$

while the trace of the energy-momentum tensor (7) vanishes at the classical level, $T_{\alpha}^{\alpha}=0$. Therefore the classical theory is invariant under the scale transformations.

However, the scale invariance is broken by quantum fluctuations. Consequently, the quantum expectation value of the right-hand side of Eq. (9) is nonzero and, consequently, on a quantum level the dilatation current (6) is no more conserved.

Consider a Weyl scale transformation of the flat metric, $\eta_{\mu \nu} \rightarrow g_{\mu \nu}(x)$, with

$$
g_{\mu \nu}(x)=e^{2 \tau(x)} \eta_{\mu \nu},
$$

For small scale factor $\tau(x)$ with $|\tau(x)| \ll 1$ the metric perturbation is $\delta g_{\mu \nu}(x)=2 \tau(x) \eta_{\mu \nu}$ and Eq. (8) implies:

$$
S \rightarrow S_{\tau}=S+\int d^{4} x \tau(x) T_{\alpha}^{\alpha}(x)+O\left(\tau^{2}\right),
$$

where $S_{\tau}$ is the action (8) of the theory (5) in a background of the rescaled flat metrics $(10)$. Therefore the expectation value of the trace of the energy-momentum tensor is given by the functional derivative

$$
\left\langle T_{\alpha}^{\alpha}(x)\right\rangle=\frac{1}{i} \frac{1}{\mathcal{Z}\left[A^{\mathrm{cl}}, \tau\right]} \frac{\delta \mathcal{Z}\left[A^{\mathrm{cl}}, \tau\right]}{\delta \tau(x)}
$$

of the generating functional

$$
\mathcal{Z}\left[A_{\mathrm{cl}}, \tau\right]=\int \mathcal{D} A \mathcal{D} \bar{\psi} \mathcal{D} \psi e^{i S_{\tau}\left[A+A^{\mathrm{cl}} ; \bar{\psi}, \psi\right]},
$$

where we have also coupled our system to a background of the classical electromagnetic field $A_{\mu}^{\mathrm{cl}}=A_{\mu}^{\mathrm{cl}}(x)$. The latter allows us to express the electric (vector) current of the fermions,

$$
j^{\mu}(x) \equiv j_{V}^{\mu}(x)=e \bar{\psi}(x) \gamma^{\mu} \psi(x),
$$

in terms of a functional derivative

$$
\left\langle j^{\mu}(x)\right\rangle=i \frac{1}{\mathcal{Z}\left[A^{\mathrm{cl}}, \tau\right]} \frac{\delta \mathcal{Z}\left[A^{\mathrm{cl}}, \tau\right]}{\delta A_{\mu}^{\mathrm{cl}}(x)} .
$$

The scale invariance should generally be broken at the quantum level because the (dimensionless) gauge coupling $e=e(\mu)$ is a function of the (dimensionful) renormalization scale $\mu$. The Weyl scale transformation (10) changes the renormalization scale, $\mu \rightarrow \mu+\delta \mu$ with $\delta \mu=\mu \delta \tau$ and, consequently, shifts the coupling $e \rightarrow e+\delta e$ by $\delta e=\beta(e) \delta \tau$ where

$$
\beta(g)=\frac{\mathrm{d}}{\mathrm{d} \ln \mu} \frac{e^{2}(\mu)}{4 \pi},
$$

is the $\beta$ function of the theory. Therefore at the quantum level the trace of the energy-momentum tensor is nonzerd ${ }^{2}$

$$
\left\langle T_{\alpha}^{\alpha}(x)\right\rangle=\frac{\beta(e)}{2 e} F_{\mu \nu}(x) F^{\mu \nu}(x),
$$

and the dilatation current $\sqrt{67}$ is no more conserved according to Eq. (9). In Eq. (17) the field-strength tensor $F_{\mu \nu}$ corresponds to the external background field $A_{\mu}^{\mathrm{cl}}$ (for the sake of simplicity we omit hereafter the superscript "cl" which refers to the classical background field).

Various aspects of conformal anomalies in hydrodynamics were discussed in Ref. [13. Below we show that the scale anomaly (17) leads to an unexpected (anomalous) contribution to electric current induced by external electromagnetic fields in spatially inhomogeneous or inflating or deflating gravitational backgrounds associated with dilatational perturbations of the form (10). In order to demonstrate the essence of the effect we consider the system at zero temperature and zero density so that both usual and chiral chemical potentials are zero, $\mu_{V}=\mu_{A}=0$. In our derivation we follow the logic of Ref. 14] which we apply to the case of the scale anomaly in the coordinate space.

The electric current $\langle j\rangle$ induced by weak external electromagnetic field $A_{\mu}(x)$ and by small local dilatations of the metric $\tau(x)$ can be expanded in series over these perturbations:

$$
\left\langle j^{\mu}\right\rangle=\left\langle j^{\mu}\right\rangle_{\text {Kubo }}+\left\langle j^{\mu}\right\rangle_{\text {dilat }}+\left\langle j^{\mu}\right\rangle_{\text {scale }}+\ldots
$$

The terms in Eq. (18) are proportional, respectively, to the first powers of $A$ and $\tau$, and to their product $A \tau$. All higher-order terms are denoted by the ellipses.

The first term in Eq. (18) is given by the standard, linear-response Kubo formula

$$
\left\langle j^{\mu}(x)\right\rangle_{\text {Kubo }}=-i \int d^{4} y \Pi^{\mu \nu}(x, y) A_{\nu}(y),
$$

where

$$
\Pi^{\mu \nu}(x, y)=\left\langle j^{\mu}(x) j^{\nu}(y)\right\rangle_{0},
$$

2 In contrast to the axial anomaly (1) the scale anomaly 17 is not a pure one-loop result. Consequently, multiloop corrections may appear in Eq. [17, see Ref. [3] for detailed discussion. 
is a two-point correlation function of electric currents. The subscript 0 in $\langle\ldots\rangle_{0}$ indicates that the expectation value 200 is calculated in a flat Minkowski space-time in the absence of external perturbations $\left(A_{\mu}=0, \delta g_{\mu \nu}=0\right)$.

The second term in Eq. (18) corresponds to a linear response of the current to the pure dilatation (11),

$$
\left\langle j^{\mu}(x)\right\rangle_{\mathrm{dilat}}=i \int d^{4} y \Pi_{D}^{\mu}(x, y) \tau(y),
$$

where

$$
\Pi_{D}^{\mu}(x, y)=\left\langle j^{\mu}(x) T_{\alpha}^{\alpha}(y)\right\rangle_{0},
$$

is a two-point correlation function of the electric current (14) and the trace of the energy-momentum tensor 77). The correlation function $(22)$ can be calculated by varying the anomalous expectation value (17) with respect to external electric field $A_{\mu}$ in a manner of Eq. 15), and setting $A_{\mu}=0$ after the variation. Since the anomaly $(17)$ is quadratic in gauge field $A_{\mu}$ the correlation function 22 is zero. Therefore the electric current (21), induced by the dilatation, is vanishing in the linear response approximation, $\left\langle j^{\mu}(x)\right\rangle_{\text {dilat }} \equiv 0$.

In our Letter we are mainly interested in the third term in Eq. (18). This term describes a scale-anomalous contribution to the expectation value of the electric current. It corresponds to a mixed gauge-gravitational response in the double-linear approximation that includes one power of the electromagnetic potential $A_{\mu}$ and one power of the scale factor $\tau$. According to Eq. 19

$$
\left\langle j^{\mu}(x)\right\rangle_{\text {scale }}=\int d^{4} y \int d^{4} z \Pi_{D}^{\mu \nu}(x, y ; z) A_{\nu}(y) \tau(z),
$$

where the three-point function

$$
\Pi_{D}^{\mu \nu}(x, y ; z)=\left\langle j^{\mu}(x) j^{\nu}(y) T_{\alpha}^{\alpha}(z)\right\rangle_{0},
$$

can be evaluated by applying twice a functional differentiation with respect to the background gauge field $A_{\mu}$ to the right-hand side of the scale anomaly relation (17):

$$
\begin{gathered}
\Pi_{D}^{\mu \nu}(x, y ; z)=-\left.\frac{\delta^{2}\left\langle T_{\alpha}^{\alpha}(z)\right\rangle}{\delta A_{\mu}(x) \delta A_{\nu}(y)}\right|_{\substack{A_{\mu} \rightarrow 0 \\
g_{\mu \nu} \rightarrow \eta_{\mu \nu}}} \\
=-\frac{2 \beta(e)}{e}\left(\eta^{\mu \nu} \eta^{\alpha \beta}-\eta^{\mu \beta} \eta^{\nu \alpha}\right) \frac{\partial^{2} \delta(x-z) \delta(y-z)}{\partial x^{\alpha} \partial y^{\beta}} .
\end{gathered}
$$

Substituting Eq. 25) into Eq. (23) one gets the anomalous electric current generated by the scale anomaly (17) in the presence of both the scale dilatation $\tau$ of the metric 100 and the background electromagnetic field $A^{\mu}$ :

$$
\left\langle j^{\mu}(x)\right\rangle_{\text {scale }}=\frac{2 \beta(e)}{e}\left[-F^{\mu \nu}(x) \partial_{\nu} \tau(x)+\tau(x) j_{\mathrm{cl}}^{\mu}(x)\right] .
$$

The first term in Eq. (26) is proportional to the electromagnetic field $F^{\mu \nu}$, which is induced by the classical electric current $j_{\mathrm{cl}}^{\mu}=-\partial_{\nu} F^{\mu \nu}$. The classical current makes the local contribution to the anomalous electric current given in the second term of Eq. 26. The presence of both terms guarantees that the anomalously generated current 26] is conserved: $\partial_{\mu}\left\langle j^{\mu}(x)\right\rangle_{\text {scale }}=0$.

Our result $(26)$ is obtained via the three-point function (24) which is defined and calculated in the flat Minkowski space-time. Thus we do not expect a metric-dependent renormalization of the current (26) in the adopted linear order in gravitational perturbation. The same property is shared by a contribution to the chiral vortical effect coming from the axial-gravitational anomaly [10], which can also be calculated in the flat space in a linear-response approximation in metric $g_{0 i}$.

We are interested in properties of the anomalous electric current far from the classical sources. Therefore, setting the classical current to zero in the region of the dilatation, $j_{\mathrm{cl}}^{\mu}=0$, we get, from Eq. (26),

$$
\left\langle j^{\mu}(x)\right\rangle_{\text {scale }}=-\frac{2 \beta(e)}{e} F^{\mu \nu}(x) \partial_{\nu} \tau(x) .
$$

In components, the anomalous current and the anomalous charge generated by the scale anomaly (27) in the background of the electric field $\boldsymbol{E}$ and the magnetic field $\boldsymbol{B}$ are, respectively, as follows:

$$
\begin{aligned}
\langle\boldsymbol{j}(x)\rangle_{\text {scale }} & =\sigma(x) \boldsymbol{E}(x)+\boldsymbol{F}(x) \times \boldsymbol{B}(x), \\
\left\langle j^{0}(x)\right\rangle_{\text {scale }} & =\boldsymbol{F}(x) \cdot \boldsymbol{E}(x),
\end{aligned}
$$

where

$$
\begin{aligned}
\sigma(t, \boldsymbol{x}) & =-\frac{2 \beta(e)}{e} \frac{\partial \tau(t, \boldsymbol{x})}{\partial t}, \\
\boldsymbol{F}(t, \boldsymbol{x}) & =\frac{2 \beta(e)}{e} \boldsymbol{\nabla} \tau(t, \boldsymbol{x}),
\end{aligned}
$$

and $\tau(x)$ is the local scale factor of the flat metric $(10)$.

The scalar quantity $\sigma$, given by Eq. (30) plays a role of an anomalous Ohm's conductivity. Indeed, in a spatially uniform $(\boldsymbol{\nabla} \tau \equiv 0)$ background $g_{\mu \nu}=e^{2 \tau(t)} \eta_{\mu \nu}$ with a time-dependent scale factor $\tau=\tau(t)$ the scale anomaly generates the anomalous electric current $(28)$ which takes precisely the functional form of Ohm's law:

$$
\langle\boldsymbol{j}(t, \boldsymbol{x})\rangle_{\text {scale }}=\sigma(t) \boldsymbol{E}(t, \boldsymbol{x}) \quad \text { for } \quad \boldsymbol{\nabla} \tau=0 .
$$

Equations (30) and 32 describe the scale electric effect (SEE): the scale anomaly generates the local electric current in the background of the external electric field in a space-time with a time-dependent scale factor.

The SEE (32) emerges in an open, expanding (or contracting) system which has an explicit arrow of time. Consequently, the SEE does not conserve entropy and does not, in general, describe a dissipationless phenomenon contrary to the chiral anomalous transport effects 22 and (3). The power $P=\langle\boldsymbol{j}\rangle_{\text {scale }} \cdot \boldsymbol{E}=\sigma E^{2}$ dissipated by the anomalous electric current (32) per unit volume may take both positive and negative values because the anomalous conductivity $(30)$ may be both a positive and negative quantity, respectively. As a result, 
in this open system the scale electric effect 32 may not only heat the system but it may also cool it by absorbing heat. We illustrate the SEE (32) in Fig. 1 .

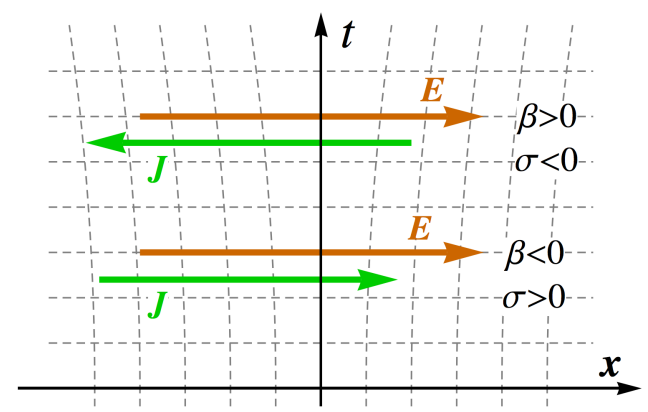

FIG. 1. The scale electric effect (SEE): in an inflating $\left(\partial_{t} \tau>0\right)$ gravitational background 10 the scale (conformal) anomaly (17) generates the electric current $\boldsymbol{J}$ along the electric field $\boldsymbol{E}$ according to Eqs. (32) and 30 . The direction of the current depends on the sign of the $\beta$ function.

The anomalous current (28) has also a contribution coming from the magnetic field $\boldsymbol{B}$. This part may only appear due to local spatial inhomogeneities of the scale factor $\tau(x)$, which are encoded in the vector quantity $\boldsymbol{F}$ in Eq. (31). According to Eqs. (28) and (31), in a nonuniformly stretched static space-time the scale anomaly generates the electric current which is transversal both to the direction of magnetic field $\boldsymbol{B}$ and to the gradient of the spatial inhomogeneity $\boldsymbol{F}$ :

$$
\langle\boldsymbol{j}(t, \boldsymbol{x})\rangle_{\text {scale }}=\boldsymbol{F}(\boldsymbol{x}) \times \boldsymbol{B}(t, \boldsymbol{x}) \text { for } \partial_{t} \tau=0 .
$$

Equations (31) and (33) describe the scale magnetic effect (SME): the scale anomaly generates the local electric current in background of external magnetic field in a spacetime with a spatially dependent scale factor (Fig. 2).

The electric current generated by the scale magnetic effect (33) flows without dissipation similarly to the current (2) generated by the chiral magnetic effect. However, there are major differences between the SME (33) and the axial-anomalous transport effects (2): (i) the scale anomaly generates the electric current via the SME in the vacuum state while the CME is realized in matter only; (ii) the SME electric current (33) is transverse to the direction of magnetic field while in the CME the magnetic field and the current are parallel to each other.

In the presence of external electric field the scale anomaly should also lead to concentration of electric charge $29 p$ at spatial inhomogeneities of the metric (31).

Using a one-loop QED $\beta$ function for one species of a Dirac fermion 5 , $\beta_{\text {QED }}^{1 \text {-loop }}(e)=e^{3} /\left(12 \pi^{2}\right)$ we get the anomalous transport coefficients (30) and (31):

$$
\sigma=-\frac{e^{2}}{6 \pi^{2}} \frac{\partial \tau}{\partial t}, \quad \boldsymbol{F}=\frac{e^{2}}{6 \pi^{2}} \nabla \tau,
$$

where $\tau(x)$ is the local scale factor of the flat metric 10 .
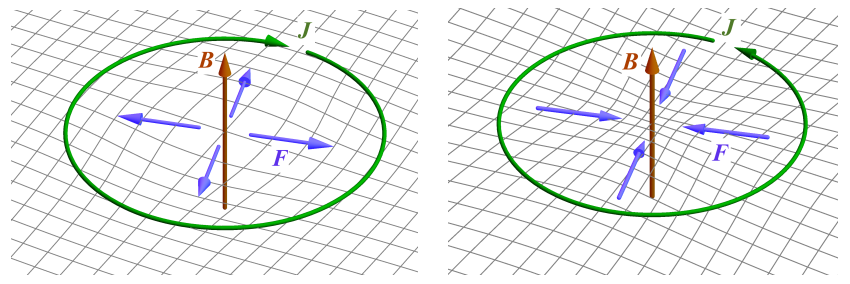

FIG. 2. The scale magnetic effect (SME): in a spatially nonuniform gravitational background the scale (conformal) anomaly (17) generates the electric current $\boldsymbol{J}$ circumnavigating the external magnetic field $\boldsymbol{B}$ according to Eq. (33). The gradient of the scale factor $\boldsymbol{F}$ is given in Eq. (31).

In an expanding geometry with a generic homogeneous isotropic metric $d s^{2}=d t^{2}-a^{2}(t) d \boldsymbol{x}^{2}$ the conductivity of each species of massless charged fermions gets an anomalous contribution (we restore $\hbar$ and $c$ ):

$$
\sigma_{\mathrm{QED}}(t)=-\frac{e^{2} H(t)}{6 \pi^{2} \hbar c},
$$

where $H(t)=\dot{a}(t) / a(t)$ is the Hubble parameter. The derivation of Eq. (35) assumes that the scale factor $\tau$ is small so that the scale factor $a(t)$ is close to unity.

Equation (35) implies that the inflating vacuum of massless fermions should have - due to the scale anomaly (17) - a nonzero negative conductivity in theories with positive beta functions, $\beta>0$. This conclusion agrees with the findings of Refs. [17] and 18, where the electric conductivity induced by, respectively, fermionic and bosonic Schwinger effects, was calculated in inflating (de Sitter) space-time. In particular, in a weak-field limit, $|e E| \ll H^{2}$, the leading term in the fermionic Schwinger effect [17] is 4 times bigger than its bosonic counterpart [18] in agreement with the relation $\beta_{\mathrm{QED}}^{1 \text {-loop }}=4 \beta_{\mathrm{SQED}}^{1 \text {-loop }}$ between usual and scalar QED beta functions ${ }^{3}$ Thus, in the particular case of homogeneous and isotropic inflation the scale-anomalous conductivity can be associated with the Schwinger pair production.

The sign of the Lorentz invariant $F_{\mu \nu} F^{\mu \nu} \propto B^{2}-E^{2}$ determines whether the electromagnetic background is magnetically $(|B|>|E|)$ or electrically $(|E|>|B|)$ dominated. In the former (latter) case the pure SME (SEE) is realized in the reference frame in which the electric (magnetic) field vanishes. In a general frame both effects are

\footnotetext{
3 The field theory situation is still somewhat unclear since fermionic and bosonic conductivities induced by the Schwinger effect [17, 18] contain explicit contributions from our generic conformal $(m=0)$ formula 30 up to a common logarithmic prefactor $\log \left(H^{2} / \mathrm{m}^{2}\right)$. The existence of this infrared-divergent factor is questioned for the massless case $(m=0)$ where the adiabatic regularization of [17, 18] may not be applicable 18] (in exactly conformal case no infrared divergences are expected to arise [21]).
} 
present, and the induced current is given by Eqs. 28(31). The magnetic field dominance is required for the existence of a stable vacuum.

Summarizing, we have shown that in theories with electrically charged massless particles the scale anomaly leads to new transport effects: the scale electric effect (SEE) and the scale magnetic effect (SME) given by Eqs. (32) and (33), respectively. The SEE implies that in inflating geometry the QED scale anomaly generates electric current 32 flowing in the opposite direction to the electric field, thus exhibiting a negative conductivity, Eq. (35). The SME implies that in a static but spatially inhomogeneous conformal gravitational background the dissipationless electric current flows transversely both to the magnetic field axis and to the gradient of the inhomogeneity, Eq. 33. The generated electric currents are proportional to the appropriate $\beta$ function. One can expect that the scale-anomalous transport effects 28 (31) are quite generic phenomena because the anomalous term (17) - which is our starting point - is present in wide varieties of physical models involving fermionic and/or bosonic degrees of freedom. They may also presumably be realized in solid state materials possessing relativistic quasiparticles, such as strained graphene [22] or elastically deformed Weyl/Dirac semimetals [23].

The author is grateful to G. Gibbons, K. Landsteiner, M. Vozmediano and M. Zubkov for interesting discussions, and to T. Kalaydzhyan, Takeshi Kobayashi, E. Megias, M. Valle and A. Vilenkin for valuable communications. The author acknowledges hospitality and support of IFT-UAM/CSIC, Madrid, as associate researcher under the Centro de Excelencia Severo Ochoa Programme Grant No. SEV-2012-0249.

* maxim.chernodub@lmpt.univ-tours.fr

[1] G. Basar and G. V. Dunne, "The Chiral Magnetic Effect and Axial Anomalies," Lect. Notes Phys. 871, 261 (2013) arXiv:1207.4199.

[2] K. Landsteiner, E. Megias and F. Pena-Benitez, "Anomalous Transport from Kubo Formulae," Lect. Notes Phys. 871, 433 (2013) arXiv:1207.5808.

[3] M. A. Shifman, "Anomalies in Gauge Theories," Phys. Rept. 209, 341 (1991)

[4] D. T. Son and A. R. Zhitnitsky, "Quantum anomalies in dense matter," Phys. Rev. D 70, 074018 (2004) hep$\mathrm{ph} / 0405216$.

[5] M. A. Metlitski and A. R. Zhitnitsky, "Anomalous axion interactions and topological currents in dense matter," Phys. Rev. D 72, 045011 (2005) hep-ph/0505072.

[6] K. Fukushima, D. E. Kharzeev and H. J. Warringa, "The Chiral Magnetic Effect," Phys. Rev. D 78, 074033 (2008) arXiv:0808.3382 [hep-ph]].

[7] A. Vilenkin, "Equilibrium Parity Violating Current In A Magnetic Field," Phys. Rev. D 22, 3080 (1980).

[8] A. Vilenkin, "Macroscopic Parity Violating Effects: Neutrino Fluxes From Rotating Black Holes And In Rotating
Thermal Radiation," Phys. Rev. D 20, 1807 (1979).

[9] D. T. Son and P. Surowka, "Hydrodynamics with Triangle Anomalies," Phys. Rev. Lett. 103, 191601 (2009) arXiv:0906.5044.

[10] K. Landsteiner, E. Megias and F. Pena-Benitez, "Gravitational Anomaly and Transport," Phys. Rev. Lett. 107, 021601 (2011) arXiv:1103.5006.

[11] G.E. Volovik, "The Universe in a Helium Droplet", Clarendon Press, Oxford (2003).

[12] D. E. Kharzeev, "Topology, magnetic field, and strongly interacting matter," Ann. Rev. Nucl. Part. Sci. 65, 193 (2015) arXiv:1501.01336; D. E. Kharzeev, J. Liao, S. A. Voloshin and G. Wang, "Chiral magnetic and vortical effects in high-energy nuclear collisions - A status report," Prog. Part. Nucl. Phys. 88, 1 (2016) arXiv:1511.04050.

[13] R. Baier, P. Romatschke, D. T. Son, A. O. Starinets and M. A. Stephanov, JHEP 0804, 100 (2008) arXiv:0712.2451; R. Loganayagam, "Entropy Current in Conformal Hydrodynamics," JHEP 0805, 087 (2008) arXiv:0801.3701; C. Eling, Y. Oz, S. Theisen and S. Yankielowicz, "Conformal Anomalies in Hydrodynamics," JHEP 1305, 037 (2013) arXiv:1301.3170; S. Bhattacharyya, "Entropy Current from Partition Function: One Example," JHEP 1407, 139 (2014) arXiv:1403.7639; E. Megias and M. Valle, "Secondorder partition function of a non-interacting chiral fluid in 3+1 dimensions," JHEP 1411, 005 (2014) arXiv:1408.0165.

[14] G. M. Newman and D. T. Son, "Response of stronglyinteracting matter to magnetic field: Some exact results," Phys. Rev. D 73, 045006 (2006) hep-ph/0510049.

[15] M. B. Fröb, J. Garriga, S. Kanno, M. Sasaki, J. Soda, T. Tanaka and A. Vilenkin, "Schwinger effect in de Sitter space," JCAP 1404, 009 (2014) arXiv:1401.4137.

[16] R. G. Cai and S. P. Kim, "One-Loop Effective Action and Schwinger Effect in (Anti-) de Sitter Space," JHEP 1409, 072 (2014) arXiv:1407.4569 [hep-th]].

[17] T. Hayashinaka, T. Fujita and J. Yokoyama, "Fermionic Schwinger effect and induced current in de Sitter space," JCAP 07, 010 (2016) arXiv:1603.04165; T. Hayashinaka and J. Yokoyama, "Point splitting renormalization of Schwinger induced current in de Sitter space-time," JCAP 07, 012 (2016) arXiv:1603.06172.

[18] T. Kobayashi and N. Afshordi, "Schwinger Effect in $4 D$ de Sitter Space and Constraints on Magnetogenesis in the Early Universe," JHEP 1410, 166 (2014) arXiv:1408.4141.

[19] C. Stahl, E. Strobel and S. S. Xue, "Fermionic current and Schwinger effect in de Sitter space-time," Phys. Rev. D 93, no. 2, 025004 (2016) arXiv:1507.01686.

[20] E. Bavarsad, C. Stahl and S. S. Xue, "Scalar current of created pairs by Schwinger mechanism in de Sitter spacetime," arXiv:1602.06556.

[21] L. Parker, S. A. Fulling, "Adiabatic regularization of the energy momentum tensor of a quantized field in homogeneous spaces," Phys. Rev. D 9, 341 (1974); S. A. Fulling, L. Parker, B. L. Hu, "Conformal energy-momentum tensor in curved space-time: Adiabatic regularization and renormalization," Phys. Rev. D 10, 3905 (1974).

[22] M. A. H. Vozmediano, M. I. Katsnelson, F. Guinea, "Gauge fields in graphene", Phys. Rep. 496, 109 (2010) arXiv:1003.5179; Fernando de Juan, Mauricio Sturla, Maria A. H. Vozmediano, "Space dependent Fermi veloc- 
ity in strained graphene", Phys. Rev. Lett. 108, 227205 (2012) arXiv:1201.2656; J. S. Bunch et al., "Impermeable Atomic Membranes from Graphene Sheets" Nano Lett., 8, 2458 (2008); G. E. Volovik and M. A. Zubkov, "Emergent Horava gravity in graphene," Annals Phys. 340, 352 (2014) arXiv:1305.4665.

[23] Onkar Parrikar, Taylor L. Hughes, and Robert G. Leigh, "Torsion, parity-odd response, and anomalies in topological states", Phys. Rev. D 90, 105004 (2014) arXiv:1407.7043. M.A.Zubkov, "Emergent gravity and chiral anomaly in Dirac semimetals in the presence of dislocations", Annals of Phys., 360, 655 (2015), arXiv:1501.04998; A. Cortijo, Y. Ferreirs, K. Landsteiner and M. A. H. Vozmediano, "Elastic Gauge Fields in Weyl Semimetals," Phys. Rev. Lett. 115, no. 17, 177202 (2015) arXiv:1603.02674; A. Cortijo and M. A. Zubkov, "Emergent gravity in the cubic tightbinding model of Weyl semimetal in the presence of elastic deformations," Annals Phys. 366, 45 (2016) arXiv:1508.04462. 